\title{
Diseño de Hardware y Software de Systems on Chip empleando tecnología Xilinx EDK
}

\author{
Julio Cadena S., Gabriel Mollocana L, Hugo Ortiz, y Vanessa Vargas V.
}

\begin{abstract}
Resumen El presente artículo resume el proceso empleado para obtener el primer System on Chip (SoC) diseñado, desarrollado, y emulado en la Escuela Politécnica del Ejército (ESPE) y en el Ecuador. Se demostrará que combinando las ventajas del diseño sobre Field Programable Gate Arrays (FPGAs) empleando la reutilización de IP Cores y plataformas, junto al uso de la tecnología de desarrollo Xilinx EDK, se puede diseñar tanto el hardware como el software de un chip de manera rápida y económicamente fiable. Además, se detalla el uso de la metodología Platform Based Design (PBD) y del concepto de co-diseño de hardware y software para diseñar las capas de hardware, sistema operativo y aplicación de un chip. La capa de hardware contiene una serie de IP Cores gobernados por un procesador MicroBlaze trabajando dentro de la arquitectura CoreConnect de IBM. Mientras que la capa de sistema operativo está conformada por drivers, librerías y el Sistema Operativo en Tiempo Real (RTOS) Xilkernel. Por último, la capa de aplicación tiene la funcionalidad de controlar una planta de temperatura, mediante la selección de dos técnicas de control: $O N$-OFF o PID. Cabe destacar que el co-diseño se desarrolló considerando un adecuado enfoque conceptual, arquitectural, y metodológico .

Palabras Claves- SoC; IP Cores; Sistemas Embebidos; MicroBlaze; RTOS.
\end{abstract}

\section{INTRODUCCIÓN}

La tendencia de la tecnología actual está basada en dispositivos electrónicos o sistemas embebidos, que posean más funciones y un mayor rendimiento, consuman menos potencia, tengan un menor tamaño y un menor precio. Además, estos sistemas deben estar disponibles lo antes posible en el mercado de consumidores. Estas características motivaron a la industria electrónica a crear una nueva metodología en el diseño de circuitos integrados. De esta manera aparecen los System on Chip (SoC).

System on chip es una tendencia a la que se le ha dado gran importancia en países que basan su economía y desarrollo en la fabricación de productos de alta tecnología. El nivel más avanzado de la tecnología en el campo de diseño de chips se ha logrado, mediante la implementación de sistemas embebidos basados en SoCs sobre Field Programmable Gate Arrays (FPGA).

Julio Cadena S., Gabriel Mollocana L., Hugo Ortiz, Vanessa Vargas V., Carrera de Ingeniería en Electrónica, Automatización y Control, Departamento de Eléctrica y Electrónica, Escuela Politécnica del Ejército, ESPE, Sangolquí, Ecuador, E-mails: juliocadena20@hotmail.com, resistron@hotmail.com, hortiz@espe.edu .ec, vcvargas@espe.edu.ec.
Los FPGAs facilitan el desarrollo de nuevos productos gracias a su reprogramabilidad en el momento mismo del diseño. La finalidad de estos dispositivos es permitir a los diseñadores de circuitos integrados plasmar sus ideas, en un menor tiempo, realizando constantes pruebas $\mathrm{y}$ cambios, hasta llegar al objetivo deseado. Para esto, los fabricantes de FPGA se esmeran en promover el diseño de herramientas que faciliten el diseño de plataformas de hardware, así como el desarrollo de herramientas para el desarrollo del software que se ejecutará sobre esta plataforma.

Cabe señalar que el desafío de los diseñadores de dispositivos electrónicos es integrar un mayor número de elementos en un simple chip, sin incrementar el tamaño del mismo, disminuyendo el tiempo de salida del producto al mercado (time-to-market) y aumentando el tiempo del producto en el mercado (time-in-market). De allí la importancia de los sistemas embebidos basados en SoCs que están diseñados para hacer alguna tarea específica, en lugar de ser un computador de propósito general para múltiples tareas.

En la actualidad, los productos basados en SoCs se encuentran abundantemente en el mercado, y van desde dispositivos portátiles, como los relojes digitales, celulares, reproductores de MP3, hasta grandes instalaciones estacionarias, luces de tráfico, controladores industriales y sistemas de control de las centrales eléctricas.

Por otra parte, el Ecuador, considerado un país en vías de desarrollo, ha estado tradicionalmente limitado en el diseño de tecnología debido a costos y a la falta de profesionales capacitados en este campo. Sin embargo con el uso de FPGAs se puede iniciar con el estudio, diseño e implementación de SoCs de forma económicamente fiable. De esta manera se augura que en un futuro cercano el Ecuador se convierta en desarrollador y exportador de tecnología.

\section{FUNDAMENTO TEÓRICO}

\section{A. System on Chip}

De acuerdo a Martin y Chang 2003, SoC es un circuito integrado complejo que integra la mayoría de elementos funcionales de un producto final completo dentro de un simple chip [1]. El uso de SoCs permite crear sistemas embebidos de menor tamaño y que incorporen mayor tecnología. La idea fundamental es convertir lo que hoy en día es un Printed Circuit Board (PCB) ${ }^{*}$ con

\footnotetext{
* Printed Circuit Board: Tarjeta de Circuitos Impresos que contiene
} 
componentes discretos en un simple SoC integrado. Tradicionalmente, diferentes componentes eran colocados e interconectados sobre una tarjeta PCB con la finalidad de cumplir una función específica (Fig. 1).

Con la utilización de Intellectual Property Cores (IP Cores), los chips individuales que conformaban los componentes en hardware fueron reemplazados por componentes virtuales, que cumplen las mismas funciones (Fig. 2). Agrupar los componentes dentro de un mismo chip disminuye notablemente el tamaño y consumo de potencia de los productos ofrecidos, obteniéndose una gran ventaja respecto a sistemas tradicionales.

Cabe señalar que los SoCs se basan en el diseño y reutilización de los bloques de propiedad intelectual IP Cores [2]. En la Fig. 3, se observa varios elementos que conforman un SoC, entre los que se destacan un procesador programable, memorias on chip, unidades de aceleración implementadas en hardware, interfaces con dispositivos periféricos, y aunque no consta en el gráfico, en un futuro podrían incluirse componentes analógicos y opto/microelectronic mechanical system (O/MEMS) [1].

\section{B. IP Cores}

Los IP Cores o Núcleos de Propiedad Intelectual son bloques con funciones preestablecidas, previamente probadas y verificadas por empresas desarrolladoras, para que posteriormente puedan ser integrados en sistemas SoC.

Una ventaja adicional de la reutilización de IP Cores, es que ofrecen una gran reducción en el riesgo de diseño de nuevos dispositivos al basarse en módulos preprobados.

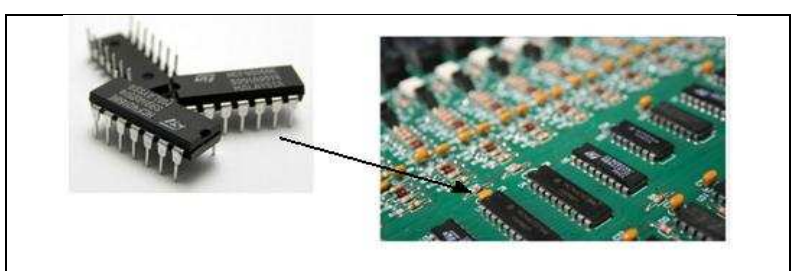

Fig. 1. Componentes Reales

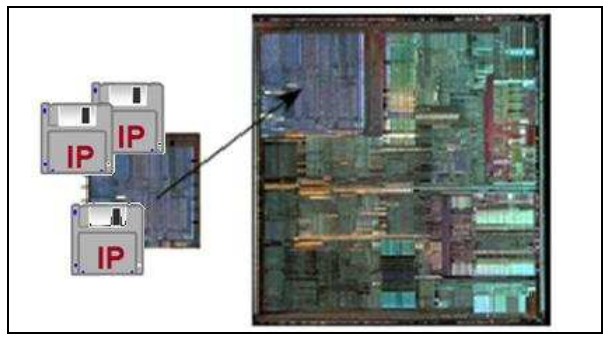

Fig. 2. Componentes Virtuales

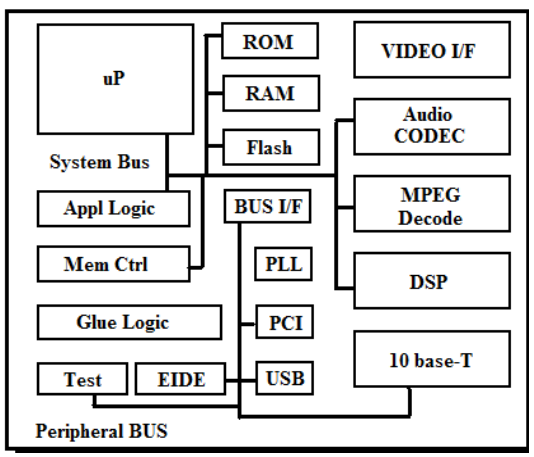

Fig. 3. System on Chip

Existen 3 tipos de IP Cores:

- Hard Cores

- $\quad$ Soft Cores

- Firm Cores

La Tabla I muestra un resumen de las características de cada tipo de IP Core.

\section{Metodologías de Diseño}

Las metodologías de diseño primarias de circuitos integrados se dividen en tres segmentos:

- Diseño Guiado por Tiempo (TDD)

- Diseño Basado en Bloques (BBD)

- Diseño Basado en Plataforma (PBD)

Los diseñadores prefieren utilizar la tecnología de Diseño Basado en Plataforma (PBD) puesto que permite disminuir el time to market expandiendo las oportunidades y la velocidad de distribución de sus productos derivados. Además, reduce varios riesgos involucrados en el diseño, facilitando la verificación de un SoC complejo debido a la gran reutilización de combinaciones de IP Cores.

Una plataforma está constituida por un conjunto de equipos y software básico, sobre el cual un grupo o familia de productos se pueden diseñar o construir gracias a que posee características comunes y una gestión integrada [1] (Fig. 4).

En la metodología PBD, el diseño agrega la reutilización de grupos de IP Cores en una arquitectura en lugar de mirar a la reutilización de IP Cores bloque por bloque [3]. La idea principal de la plataforma es simplificar el proceso de diseño.

Una vez analizados los conceptos de plataforma en la siguiente sección se va describir la plataforma utilizada en el desarrollo de este proyecto. 
TABla I. Resumen de las CARACTERÍsticAs DE IP CORES

\begin{tabular}{|c|c|c|c|}
\hline Tipo & Soft Core & Firm Core & Hard Core \\
\hline $\begin{array}{c}\text { NIVEL DE } \\
\text { ABSTACCION }\end{array}$ & $\begin{array}{c}\text { Register Transfer } \\
\text { Level (RTL), } \\
\text { gate level }\end{array}$ & $\begin{array}{c}\text { Gate level, } \\
\text { layout }\end{array}$ & Layout \\
\hline DESCRIPCION & VHDL, Verilog & Netlist $^{\text {a }}$ & $\begin{array}{c}\text { Descripción } \\
\text { de } \\
\text { transistores }\end{array}$ \\
\hline PORTABILIDAD & $\begin{array}{c}\text { A todas las } \\
\text { tecnologías }\end{array}$ & $\begin{array}{c}\text { Limitada a } \\
\text { tecnologías } \\
\text { probadas }\end{array}$ & $\begin{array}{c}\text { Optimizada } \\
\text { a una } \\
\text { tecnología } \\
\text { especifica }\end{array}$ \\
\hline FLEXIBILIDAD & Alta & Limitada & Muy poca \\
\hline PREVISIBILIDAD & Baja & Buena & $\begin{array}{c}\text { Alta y } \\
\text { definida } \\
\text { por la } \\
\text { tecnología }\end{array}$ \\
\hline $\begin{array}{c}\text { PROTECCION } \\
\text { PROPIEDAD } \\
\text { INTELECTUAL }\end{array}$ & Difícil & Fácil & Fácil \\
\hline
\end{tabular}

a. Netlist: Representación en lenguaje de descripción de hardware de la conectividad de un circuito.

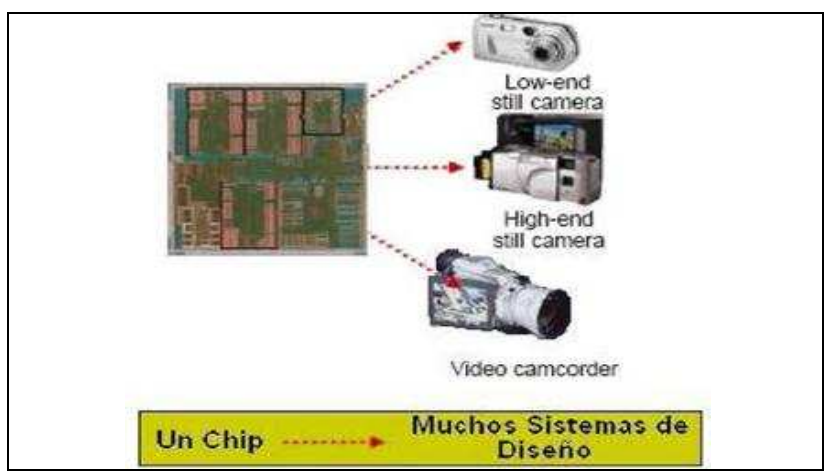

Fig. 4. Plataforma para diferentes equipos de electrónica de consumo

\section{Co-Diseño de Hardware y Software}

En el flujo o proceso de diseño convencional, grupos independientes de expertos diseñan el hardware y el software de un chip, sin que exista necesariamente cooperación entre ellos (Fig. 5a). Sin embargo en el diseño de SoCs se plantea un nuevo concepto, llamado "Co - Diseño", en el cual el chip es diseñado por grupos de expertos en cooperación (Fig. 5b).

En el Co-diseño, el hardware y el software de un sistema embebido se desarrollan en paralelo, realizando constantes realimentaciones entre los equipos de diseño. El resultado es que cada parte puede tomar ventaja de lo que la otra puede hacer. La explicación de las fases del co-diseño se realizará junto con la implementación en la sección IV.

\section{Plataforma de DeSARRollo XilinX SPARTAN-6 FPGA EMBEDDED KIT}

\section{A. Plataforma de Hardware}

El Xilinx Spartan-6 FPGA Embedded Kit tiene como tarjeta de desarrollo el modelo SP605 [4]. Esta tarjeta permite a los diseñadores de hardware y software emular sus diseños sobre el FPGA Spartan 6 LX45T (parte central de la Fig. 6). Cabe mencionar que este kit incluye toda la documentación del MicroBlaze Processor Subsystem, el cual es un SoC que puede ser utilizado como plataforma base para el desarrollo de proyectos, como es el caso del sistema implementado.

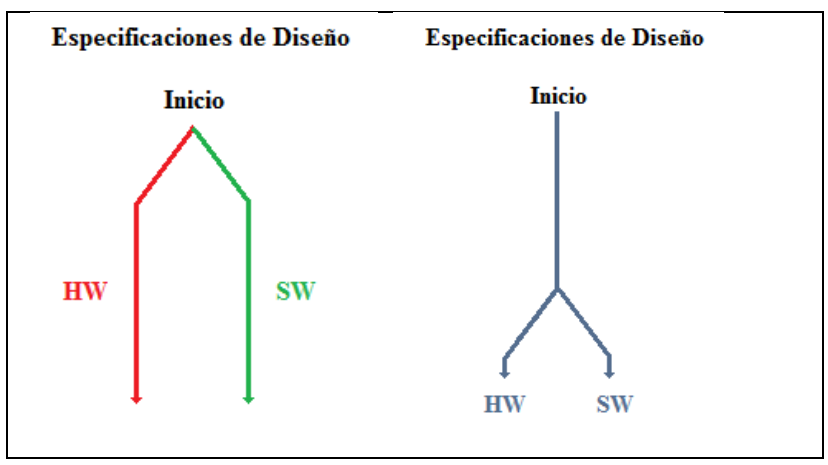

Fig. 5. (a) Flujo de Diseño Tradicional (b) Flujo de Co-Diseño

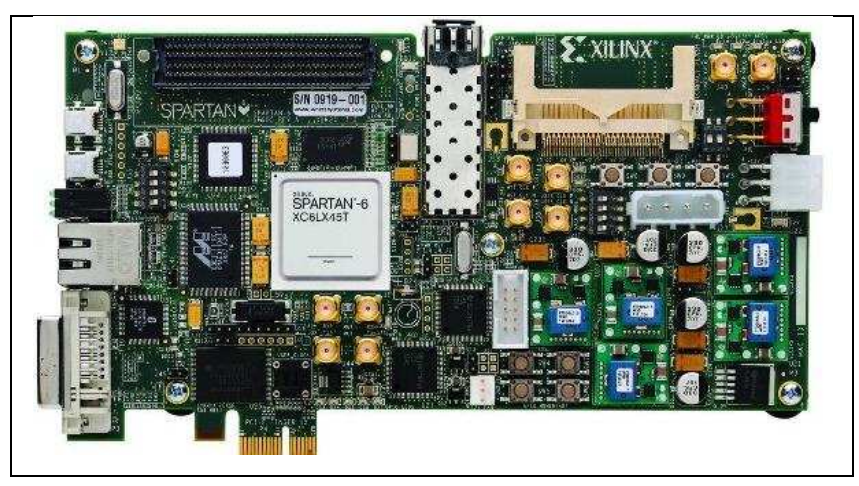

Fig. 6. Tarjeta de desarrollo SP605

\section{B. Plataforma de Software}

Está constituida por el ISE Design Suite Embedded Edition 12.1. Este software proporciona herramientas para el diseño embebido y una serie de IP Cores adaptados a las necesidades comunes de los desarrolladores. Una de sus herramientas principales es el Embedded Development Kit (EDK).

El EDK incluye Xilinx Platform Studio (XPS) (Fig. 7), para el diseño de hardware, y Software Development Kit (SDK)(Fig. 8) para el diseño de software. Así como, la documentación de la mayoría de IP Cores que se podrían necesitar en el diseño de SoCs con procesadores PowerPC y/o MicroBlaze [5 - 6]. 


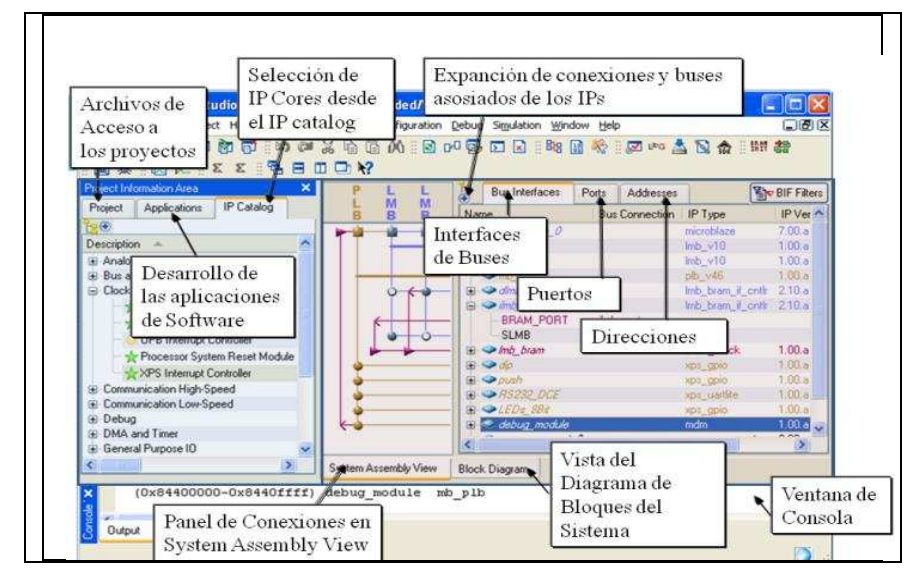

Fig. 7. Vista general del XPS

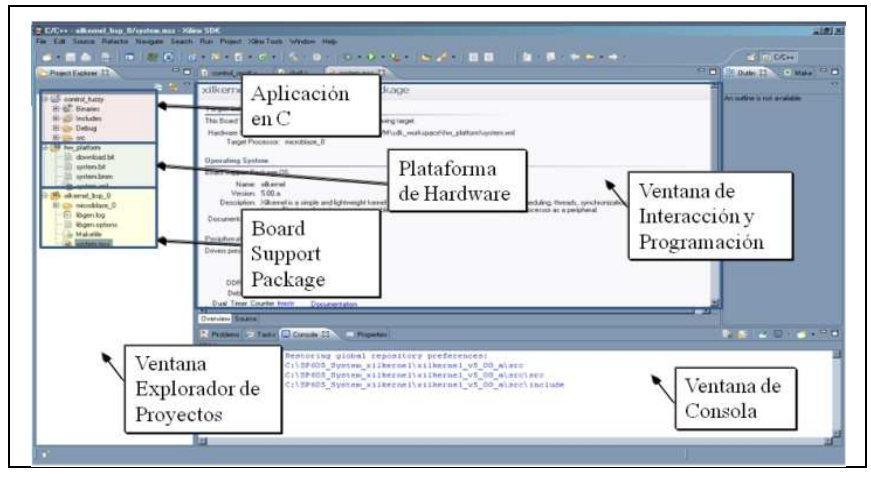

Fig. 8. Vista general del SDK

\section{IMPLEMENTACIÓN DEL CO-DISEÑO DE HARDWARE Y SOFTWARE}

El SoC diseñado en este proyecto se basa en las fases del proceso de Co-diseño, (Fig. 9) propuestas por Martin y Chang en el libro Surviving the SoC Revolution de 1999.

A continuación se detallará cada fase con los resultados obtenidos en este proyecto:

\section{A. Fase 1}

\section{1) Modelamiento Funcional}

En esta fase se establece los requerimientos del producto, $y$ se verifica las especificaciones del funcionamiento del sistema.

La planta que se desea controlar tiene las siguientes especificaciones técnicas: un rango de control de temperatura entre 40 y 65 grados centígrados, y un consumo de corriente de 1.35 [A].

El proceso de control de la planta se muestra en la Fig. 10. Donde, el elemento controlador está conformado por el SoC emulado sobre la tarjeta de desarrollo SP605.

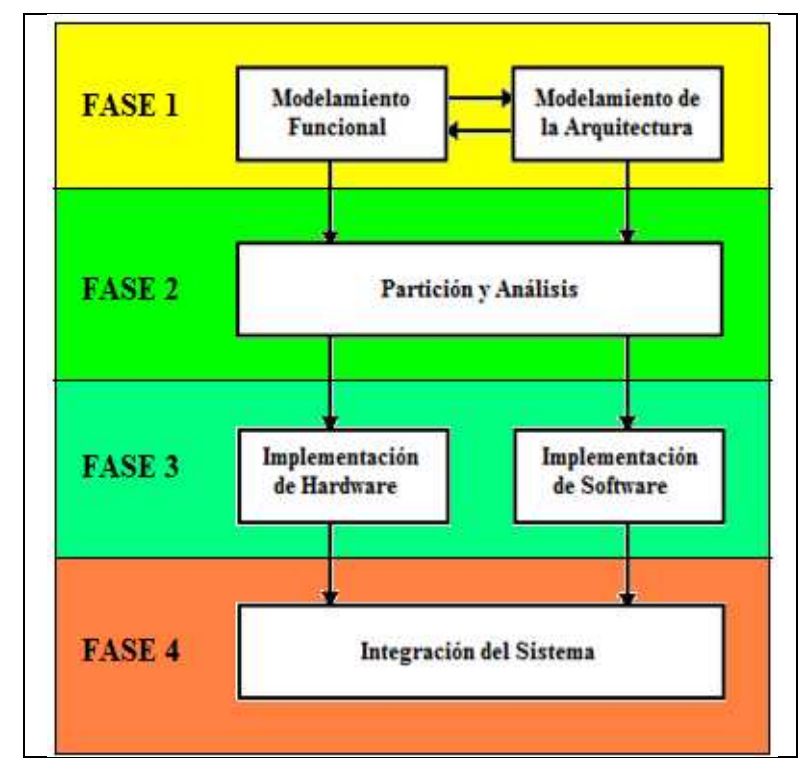

Fig. 9. Fases del Co-Diseño HW/SW

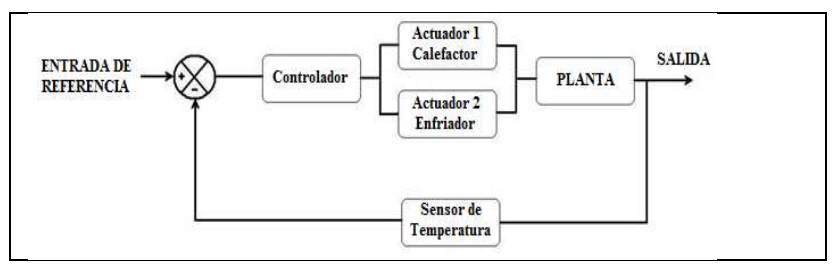

Fig. 10. Esquema de Proceso de Control para Planta de Temperatura

Dadas las especificaciones de la planta, el SoC a diseñarse requiere:

En el hardware: Un procesador, memorias, buses, y periféricos de entrada/salida de propósito general, adquisición de datos, controlador de interrupciones, módulo de depuración, interfaz de comunicación serial y soporte para un Sistema Operativo en Tiempo Real (RTOS).

En el software: Un RTOS para el trabajo con hilos, semáforos e interrupciones, drivers para dispositivos de hardware, código de rutinas e hilos para realizar el control de la planta, y una Shell CLI (Command Line Interface) que permita la selección de varios sub-programas a través del ingreso de comandos.

\section{2) Modelamiento de la Arquitectura}

Una vez que las especificaciones funcionales están definidas se procede a escoger una arquitectura que ejecute las funciones del sistema. Generalmente esta arquitectura queda definida por la plataforma que se vaya a emplear.

Tal como se señaló la plataforma de desarrollo que se utilizó es XILINX SPARTAN-6 FPGA EMBEDDED KIT. Sus herramientas facilitan la implementación de una arquitectura de hardware, basada en el estándar 
CoreConnect de IBM y en un procesador Microblaze para el procesamiento de la información y toma de decisiones. Esta arquitectura se define en el software XPS.

CoreConnect implementa un Processor Local Bus (PLB) para conectar el CPU a los periféricos, y un Local Memory Bus (LMB) para conectarlo a las memorias del sistema. Además, su topología es tipo bus, lo que significa que los IP Cores comparten una misma línea y protocolo de comunicación. La ventaja de utilizar esta topología es que si falla un elemento no genera el fallo de todo el sistema (Fig. 11).

\section{B. Fase 2: Partición y Análisis}

En esta fase se realiza una partición del modelo funcional sobre el modelo de la arquitectura. Es decir, se asigna las tareas del sistema a un recurso específico de hardware, o a un recurso de software.

El modelo funcional ha sido establecido en forma de capas, en base a las especificaciones necesarias del sistema, como se muestra en la Fig. 12.

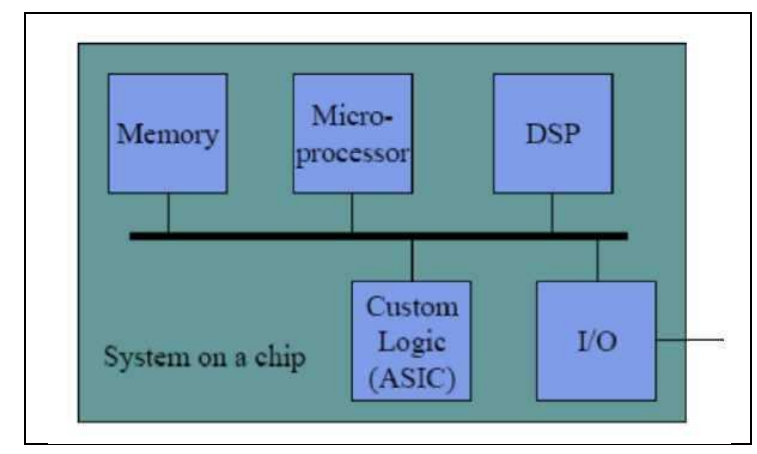

Fig. 11. SoC basado en topología BUS

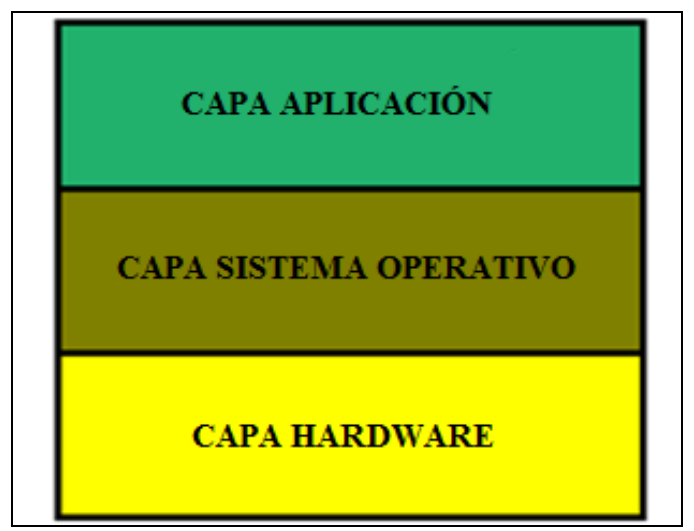

Fig. 12. Vista en Capas del Diseño

- CAPA HARDWARE: Diseño realizado en XPS de la plataforma de hardware.

- CAPA SISTEMA OPERATIVO: BSP creado en SDK de la plataforma de software.

- CAPA APLICACIÓN: Aplicación de software en lenguaje $\mathrm{C}$ desarrollada en SDK.

La Capa de Hardware ejecutará las siguientes tareas:
- Adquisición de datos del sensor de temperatura XPS ADC core.

- Manejo de actuadores de calefacción (Foco) y de enfriamiento (Ventilador) - XPS GPIO core.

- Interfaz de usuario RS232 - UART_16550_core.

- Controlador de memoria externa DDR3 - MPMC DDR3 SDRAM.

- Reloj del sistema - clock generator.

- Soporte para RTOS - XPS Timer.

- Generación de periodo de muestreo - XPS Timer.

- Controlador de interrupciones - XPS Interrup Controler.

- Depuración del sistema - Debug Module.

Por otro lado, el software del sistema contiene las capas de Sistema Operativo y de Aplicación. Las funciones que cumple la Capa de Sistema Operativo, a través de las librerías del RTOS Xilkernel son:

- Scheduling

- APIs para hilos POSIX

- APIs para semáforos

- APIs para interrupciones

- Drivers de periféricos de hardware

En tanto que a la Capa de Aplicación, realizar las siguientes rutinas de software en lenguaje C:

- Interfaz de línea de comandos (Shell CLI).

- Control de temperatura ON-OFF.

- Control de temperatura PID.

- Gestión del reloj del sistema.

- Gestión de interrupciones.

- Hardware Setup.

C. Fase 3

\section{1) Implementación de Hardware}

Esta fase abarca el diseño de nuevos bloques de hardware y la integración de bloques reusables o IP Cores. Finaliza con la síntesis del código VHDL de la plataforma de hardware resultante.

Para crear el SoC controlador se tomó como referencia la plataforma MicroBlaze Processor Subsystem, basada en el procesador MicroBlaze. De esta plataforma se eliminó los IP Cores que no eran de interés. Además, se añadió otros IP Cores para complementar el sistema y se realizó nuevas configuraciones en algunos de los ya existentes.

\section{a) Capa de Hardware}

El procedimiento de diseño de la capa de hardware empleando las herramientas XPS es el siguiente: 
- Personalización del Microblaze Processor Subsystem

- Asignación De Pines Del FPGA Spartan 6 en el Archivo UCF

- Generación del Archivo .bit (Bitstream) de la Plataforma De Hardware

- Exportación de la Plataforma de Hardware al SDK

El SoC creado tiene el diagrama de bloques (Fig. 13) expresado a partir de la vista RTL que ofrece el XPS. En este diagrama constan los IP Cores listados en la Tabla II.

El mapa de memoria a través del cual el procesador MicroBlaze, accede a los registros internos de cada uno de estos IP Cores se muestra en la Tabla III.

Los resultados de la síntesis de este hardware se muestran en la Tabla IV.

De acuerdo a los datos proporcionados por el XPS se puede acotar que el grado de utilización del FPGA Spartan 6 fue aproximadamente un $25 \%$ de su , como se observa en la Tabla V. Es decir, esta tarjeta brinda la posibilidad de agregar gran cantidad de hardware que aumente las funcionalidades del sistema.

TABLA II. IP CORES PRESENTES EN LA CAPA DE HARDWARE

\begin{tabular}{|c|c|c|}
\hline Nombre & IP Core & Versión \\
\hline Debug_Module & mdm & $1.00 . \mathrm{g}$ \\
\hline Microblaze_0 & microblaze & $7.30 . \mathrm{a}$ \\
\hline mb_plb & plb_v46 & $1.04 . \mathrm{a}$ \\
\hline Ilmb & lmb_v10 & $1.00 . \mathrm{a}$ \\
\hline Dlmb & lmb_v10 & $1.00 . \mathrm{a}$ \\
\hline LocalMemory_Cntlr_D & lmb_bram_if_cntlr & $2.10 . \mathrm{b}$ \\
\hline LocalMemory_Cntlr_I & lmb_bram_if_cntlr & $2.10 . \mathrm{b}$ \\
\hline lmb_bram & bram_block & $1.00 . \mathrm{a}$ \\
\hline RS232_Uart_1 & xps_uart16550 & $3.00 . \mathrm{a}$ \\
\hline clock_generator_0 & clock_generator & $4.00 . \mathrm{a}$ \\
\hline DDR3_SDRAM & mpmc & $6.00 . \mathrm{a}$ \\
\hline proc_sys_reset_0 & proc_sys_reset & $2.00 . \mathrm{a}$ \\
\hline Interrupt_Cntlr & xps_intc & $2.01 . \mathrm{a}$ \\
\hline timer_xilkernel & xps_timer & $1.02 . \mathrm{a}$ \\
\hline timer_clock & xps_timer & $1.02 . \mathrm{a}$ \\
\hline timer_sample & xps_timer & $1.02 . \mathrm{a}$ \\
\hline PWM & xps_timer & $1.02 . \mathrm{a}$ \\
\hline ADC & xps_deltasigma_adc & $1.01 . \mathrm{a}$ \\
\hline gpio_ventilador & xps_gpio & $2.00 . \mathrm{a}$ \\
\hline \multicolumn{2}{|c}{}
\end{tabular}

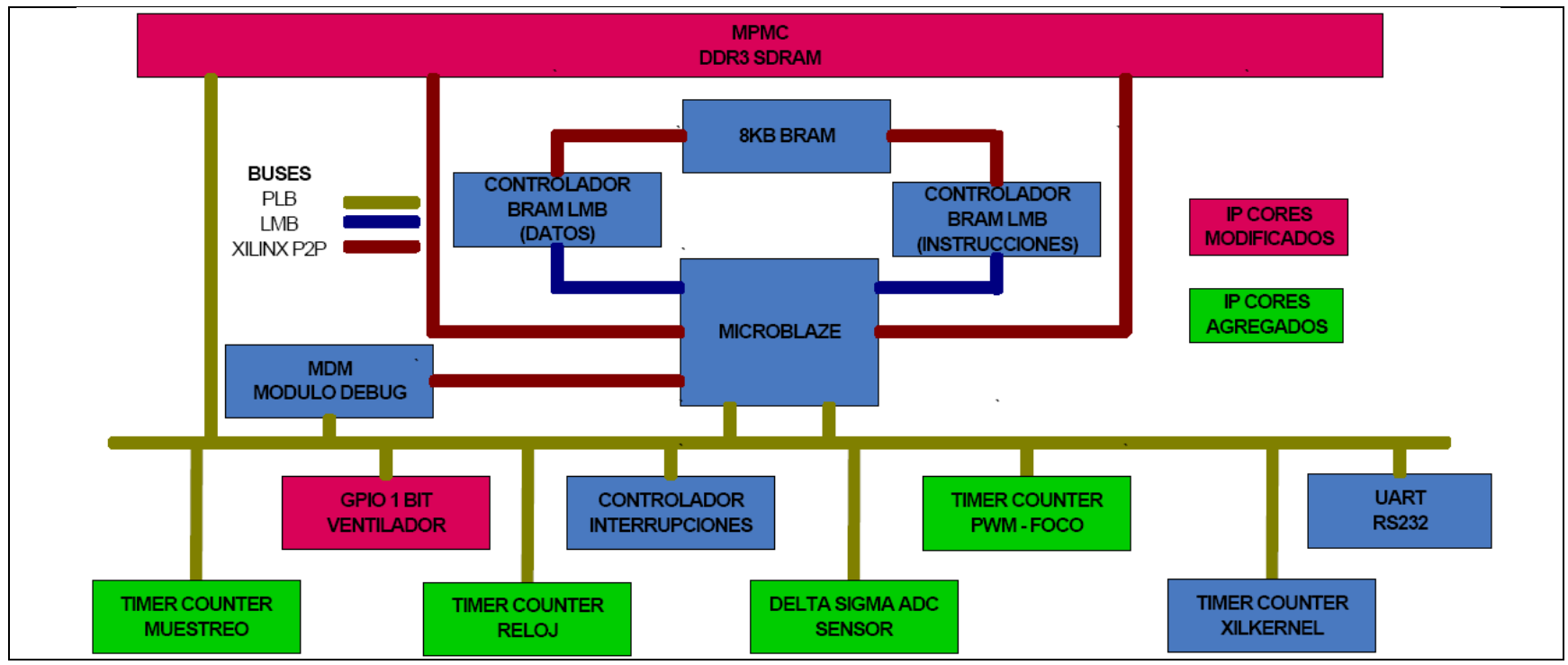

Fig. 13. Diagrama de Bloques del SoC

TABLA III. MAPA DE MEMORIA PARA MicroBlaze

\begin{tabular}{|c|c|c|}
\hline Nombre Módulo & Dirección Base & Dirección Superior \\
\hline LocalMemory_Cntlr_D & 0x00000000 & 0x00001fff \\
\hline LocalMemory_Cntlr_I & 0x00000000 & 0x00001fff \\
\hline Debug_Module & 0x84400000 & 0x8440ffff \\
\hline RS232_Uart_1 & 0x83e00000 & 0x83e0ffff \\
\hline DDR3_SDRAM & 0x88000000 & 0x8fffffff \\
\hline Interrupt_Cntlr & 0x81800000 & 0x8180ffff \\
\hline timer_xilkernel & 0x83c00000 & 0x83c0ffff \\
\hline timer_clock & 0x83c40000 & 0x83c4ffff \\
\hline timer_sample & 0x83c20000 & 0x83c2ffff \\
\hline PWM & 0x83c60000 & 0x83c6ffff \\
\hline ADC & 0x80400000 & 0x8040ffff \\
\hline Gpio_ventilador & 0x81400000 & 0x8140ffff \\
\hline
\end{tabular}

TABla IV. Resultados DE LA Síntesis DEL SoC

\begin{tabular}{|c|c|c|c|}
\hline \multicolumn{4}{|c|}{ Resumen Síntesis XPS } \\
\hline Reporte & $\begin{array}{c}\text { Uso Flip } \\
\text { Flops }\end{array}$ & $\begin{array}{c}\text { Uso } \\
\text { LUTs }\end{array}$ & $\begin{array}{c}\text { Uso } \\
\text { BRAMs }\end{array}$ \\
\hline system & 5403 & 5882 & 14 \\
\hline clock_generator_0_wrapper & & 1 & \\
\hline gpio_ventilador_wrapper & 77 & 44 & \\
\hline adc_wrapper & 217 & 183 & \\
\hline pwm_wrapper & 361 & 344 & \\
\hline timer_sampler_wrapper & 361 & 344 & \\
\hline timer_clock_wrapper & 361 & 344 & \\
\hline timer_xilkernel_wrapper & 361 & 344 & \\
\hline interrupt_cntlr_wrapper & 192 & 162 & \\
\hline proc_sys_reset_0_wrapper & 67 & 52 & \\
\hline ddr3_sdram_wrapper & 918 & 1083 & \\
\hline rs232_uart_1_wrapper & 370 & 423 & \\
\hline
\end{tabular}




\begin{tabular}{|c|c|c|c|}
\hline lmb_bram_wrapper & & & 4 \\
\hline localmemory_cntrl_i_warppe & 2 & 6 & \\
\hline localmemory_cntrl_d_warppe & 2 & 6 & \\
\hline dlmb_wrapper & 1 & 1 & \\
\hline ilmb_wrapper & 1 & 1 & \\
\hline mb_plb_wrapper & 160 & 423 & \\
\hline microblaze_0_wrapper & 1833 & 2001 & 10 \\
\hline debug_module_wrapper & 119 & 120 & \\
\hline
\end{tabular}

TABLA V. RESUMEN DE UTILIZACIÓN DEL DISPOSITIVO FPGA

\begin{tabular}{|c|c|c|c|}
\hline \multicolumn{5}{|c|}{ Resumen de Utilización del Dispositivo } \\
\hline Utilización de bloques lógicos & Uso & Disponible & Uso \\
\hline Number of Slice Registers & 4,810 & 54,576 & $8 \%$ \\
\hline Number used as Flip Flops & 4,809 & & \\
\hline Number used as Latches & 0 & & \\
\hline Number used as Latch-thrus & 0 & & \\
\hline Number used asAND/OR logics & 1 & & \\
\hline Number of Slice LUTs & 5,455 & 27,288 & $19 \%$ \\
\hline Number used as logic & 4,343 & 27,288 & $15 \%$ \\
\hline Number used as Memory & 263 & 6,408 & $4 \%$ \\
\hline Number occupied Slices & 2,076 & 6,822 & $30 \%$ \\
\hline $\begin{array}{c}\text { Number of LUT Flip Flop pairs } \\
\text { used }\end{array}$ & 5,753 & & \\
\hline Number with an unused Flip Flop & 2,167 & 5,753 & $37 \%$ \\
\hline Number with an unused LUT & 298 & 5,753 & $5 \%$ \\
\hline $\begin{array}{c}\text { Number of fully used LUT-FF } \\
\text { pairs }\end{array}$ & 3,288 & 5,753 & $57 \%$ \\
\hline Number of unique control sets & 384 & & \\
\hline $\begin{array}{c}\text { Number of slice register sites lost } \\
\text { to control set restrictions }\end{array}$ & 1,500 & 54,576 & $2 \%$ \\
\hline Number of bonded IOBs & 58 & 296 & $19 \%$ \\
\hline $\begin{array}{c}\text { Number of LOCed IOBs } \\
\text { IOB Flip Flops }\end{array}$ & 58 & 58 & 100 \\
\hline $\begin{array}{c}\text { Number of } \\
\text { BUFIO2/BUFIO2_2CLKS }\end{array}$ & 2 & 32 & $6 \%$ \\
\hline Number of RAMB16BWERS & 14 & 116 & $12 \%$ \\
\hline Number of RAMB8BWERS & 0 & 232 & $0 \%$ \\
\hline
\end{tabular}

\section{2) Implementación de Software}

En esta fase se realiza:

- La programación de la aplicación de software a través de un IDE, utilizando los drivers y librerías necesarios

- La compilación del código del programa, dado en lenguajes como $\mathrm{C}$ o $\mathrm{C}++, \mathrm{y}$

- Su almacenamiento en el núcleo del procesador.

El procedimiento de diseño empleando las herramientas SDK es el siguiente:

- Creación de un Workspace en SDK

- Importación de la Plataforma de Hardware

- Creación y Configuración del Board Support Package (BSP)

- Creación o Importación un proyecto de Software

- Descarga del archivo .bit (bitstream) de hardware al FPGA para emulación.
Los resultados en las capas Sistema Operativo y Aplicación fueron los siguientes:

\section{a) Capa Sistema Operativo}

El Board Support Package de esta capa contiene los drivers y librerías para el manejo de las funciones de hardware, y el RTOS Xilkernel para el trabajo con hilos, semáforos e interrupciones.

El sistema operativo es el xilkernel versión 5.00.a. En tanto que los drivers de los dispositivos periféricos del BSP se enlistan a continuación:

- $\mathrm{ADC}$ (dsadc)

- DDR3_SDRAM (mpmc)

- Debug_Module (uartlite)

- Interrup_Cntlr (intc)

- LocalMemory_Cntlr_D (bram)

- LocalMemory_Cntlr_I (bram)

- PWM (tmrctr)

- RS232_Uart_1 (uartns550)

- gpio_ventilador (gpio)

- timer_clock (tmrctr)

- timer_sample (tmrctr)

- timer_xilkernel (tmrctr).

La configuración de los módulos de Xilkernel se define a través de parámetros en el archivo system.mss del proyecto.

\section{b) Capa Aplicación}

En la Capa de Aplicación del sistema se desarrolló un proyecto de software que contiene los ficheros que se describen en la Tabla VI. El fichero Shell.c contiene una Shell CLI que ejecuta varios comandos para interactuar con el usuario. Además permite enlistar y ejecutar hilos previamente probados al sistema. En este caso se enlistó los hilos de control On-Off y PID a la aplicación que se ejecutan con los comandos run 0 y run 1 respectivamente.

\section{Fase 4: Integración del Sistema}

Con el hardware y software desarrollados, se procede a ensamblar el sistema completo para la realización de pruebas de laboratorio. El FPGA Spartan 6 de la tarjeta SP605 fue configurado con el bitstream de hardware generado en XPS y el código de software creado en SDK.

La implementación de un producto final suele incluir emuladores y prototipos rápidos antes de la fabricación en serie, para verificar las funciones de hardware y software del sistema. En este proyecto se llega únicamente hasta la emulación del producto sobre la tarjeta SP605. 
La Fig. 14 muestra en un diagrama de bloques la disposición física de elementos de la sistema completo.

TABLA VI. DESCRIPCIÓN DEL PROYECTO DE LA CAPA DE APLICACIÓN

\begin{tabular}{|c|c|}
\hline ARCHIVO & DESCRIPCION \\
\hline shell.c & $\begin{array}{l}\text { Este código implementa una Shell CLI que } \\
\text { contiene comandos básicos para interactuar con } \\
\text { el sistema operativo, es capaz de cargar y } \\
\text { ejecutar nuevos hilos. Se le ha cargado los hilos } \\
\text { para control on-off y PID. }\end{array}$ \\
\hline clock.c & $\begin{array}{l}\text { Implementa un hilo que sirve de reloj del } \\
\text { sistema capaz de ser seteado. Se ejecuta } \\
\text { siempre en paralelo al resto de hilos. }\end{array}$ \\
\hline control_on_off.c & $\begin{array}{l}\text { Contiene el hilo para control on-off de } \\
\text { temperatura y su bucle de control. Se lo ejecuta } \\
\text { a través del ingreso del comando "run 0" en la } \\
\text { Shell, y termina con la interrupción externa del } \\
\text { pulsador de la tarjeta SP605. }\end{array}$ \\
\hline control_pid.c & $\begin{array}{l}\text { Contiene el hilo para control PID de } \\
\text { temperatura y su bucle de control. Se lo ejecuta } \\
\text { a través del ingreso del comando "run 1" en la } \\
\text { Shell, y termina con la interrupción externa del } \\
\text { pulsador de la tarjeta SP605. }\end{array}$ \\
\hline control_header.c & $\begin{array}{l}\text { Contiene funciones para realizar setup de } \\
\text { hardware, lectura del sensor a través del ADC, } \\
\text { inicializar el timer de muestreo y para el } \\
\text { ingreso de números enteros. Estas funciones } \\
\text { son usadas en diversas partes del código por lo } \\
\text { que conforman una librería extra, asociada a la } \\
\text { aplicación a través del archivo de cabecera } \\
\text { "control_header.h" }\end{array}$ \\
\hline control_header.h & $\begin{array}{l}\text { Archivo de cabecera que contiene los } \\
\text { prototipos de las funciones del archivo } \\
\text { control_header.c, además de definiciones } \\
\text { generales de la aplicación. }\end{array}$ \\
\hline Lscript.ld & $\begin{array}{l}\text { Linker Script de la aplicación. Asocia todas las } \\
\text { secciones del programa a la memoria DDR3 } \\
\text { externa de la tarjeta SP605. Define un tamaño } \\
\text { de pila (stack size) de } 2 \mathrm{~Kb} \text { necesario para } \\
\text { ejecutar los hilos de control. }\end{array}$ \\
\hline
\end{tabular}

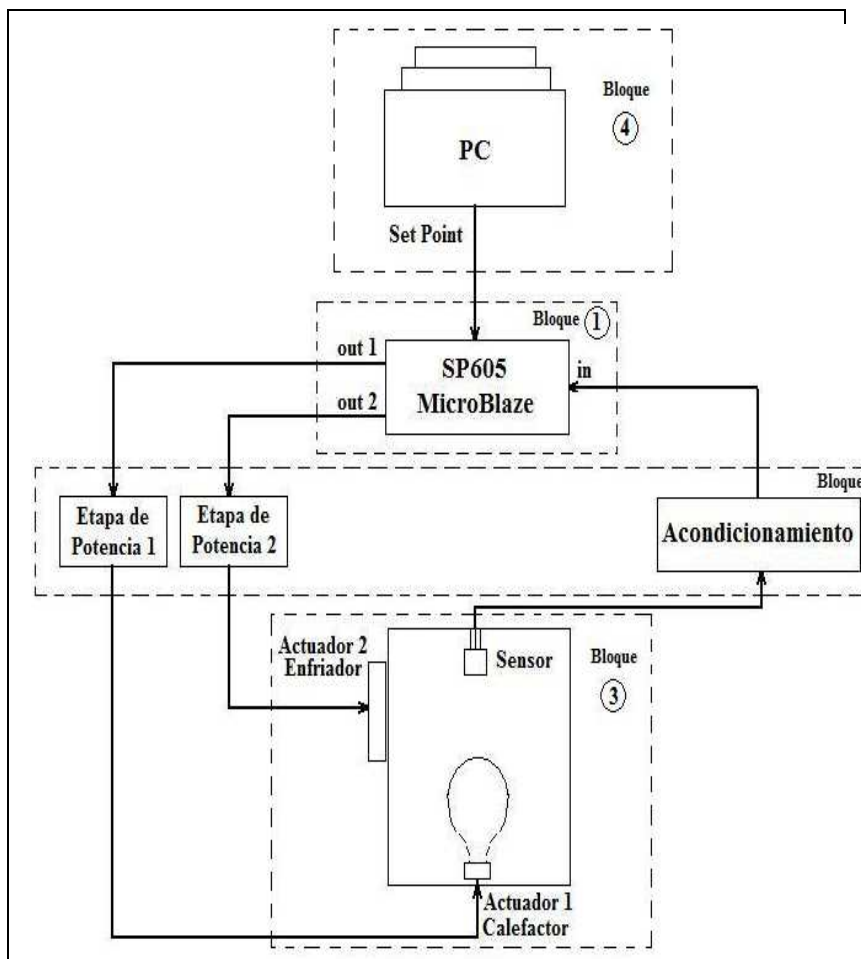

Fig. 14. Disposición Física de Elementos de la Aplicación

\section{1) Descripción de Bloques}

a) Bloque 1.- Este bloque corresponde a la plataforma de emulación SP605, sobre la cual se encuentra el SoC controlador que ha cumplido con las especificaciones del sistema.

b) Bloque 2.- Este bloque corresponde a la tarjeta de acondicionamiento de entradas y salidas, que contendrá los circuitos necesarios para trabajar con la planta de temperatura y la tarjeta SP605. Los circuitos externos implementados fueron:

- Dos etapas de potencia para los actuadores.

- Acondicionamiento para el sensor de la planta.

- Hardware externo necesario para trabajar con los IP Cores del SoC.

c) Bloque 3.- Corresponde a la planta.

d) Bloque 4.- Consiste de una terminal RS232 que sirva como interfaz de usuario, y permita el ingreso de comandos y la visualización de resultados. Se puede utilizar la consola del SDK o la hiperterminal de Windows para este propósito.

\section{2) Ejecución del Sistema Integrado}

Para ejecutar el sistema se debe configurar el FPGA con el bitstream de hardware y cargar el código del programa en la memoria. En la terminal RS232 se observará lo siguiente: 


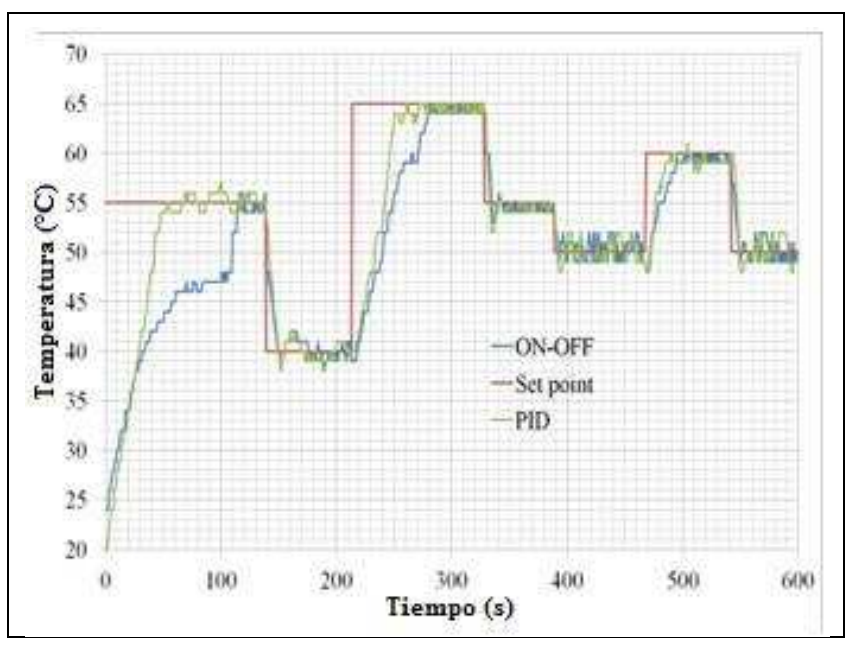

Fig. 15. Resultado control On-Off y control PID

SETUP: Plataforma de hardware inicializada
correctamente.

Iniciando Xilkernel...

SHELL: Xilkernel inicializado

SHELL: Inicializando reloj...

RELOJ: Registrado gestor de interrupciones para el timer del reloj.

RELOJ: Configurando timer del reloj para generar interrupciones cada segundo ..

RELOJ: Interrupcion de reloj habilitada ...

shell> _

Para ejecutar las rutinas de control On-Off y PID se ingresa los comandos run 0 y run 1 respectivamente. Los resultados del control On-Off y del control PID se muestran en la Fig. 15.

Es importante señalar que si bien los resultados de ambos tipos de control son aceptables, se debería trabajar en la sintonización del controlador PID. Cabe recalcar que no se puso énfasis en esta parte puesto que el objetivo principal de este proyecto fue el crear un SoC bajo el enfoque de la metodología PBD. La aplicación de control de la planta de temperatura es solo un ejemplo de la capacidad y flexibilidad que se tiene al realizar el codiseño de hardware y software.

\section{CONCLUSIONES}

El objetivo de crear un System on Chip (SoC) orientado a una aplicación de automatización y control que incluye un Sistema Operativo en Tiempo Real (RTOS), mediante el co-diseño de hardware y software fue cumplido a cabalidad.

La plataforma del sistema de control diseñado es fácilmente escalable para el desarrollo de nuevas aplicaciones de control.

La versatilidad que ofrece diseñar sobre Field Programmable Gate Array (FPGA) debe ser aprovechada en el diseño de sistemas que satisfagan las necesidades de la sociedad ecuatoriana.

Se demostró que el concepto de co-diseño y el uso FPGAs permiten al diseñador intervenir en la implementación de cada capa de un sistema embebido (hardware, sistema operativo, y aplicación). Esta característica permite realizar optimizaciones en cualquier capa y en cualquier momento del diseño, de forma económicamente fiable.

Por otro lado, se concluye que las herramientas de diseño con altas prestaciones, como las de Xilinx, constituyen un factor clave en la rápida implementación de SoCs. La ayuda que brindan al generar automáticamente la arquitectura de hardware, el mapa de direcciones, la asignación de pines, el Board Support Package (BSP) y los test de memorias y periféricos, disminuye enormemente la complejidad de un diseño.

Por último, se concluye que el presente proyecto fue el primer paso del Departamento de Eléctrica y Electrónica (DEEE) en la incursión hacia un nuevo modelo de negocios, que se basa en el desarrollo y exportación de tecnología SoC. Dentro de este ámbito se encuentran empresas desarrolladoras de IP Cores, arquitecturas y plataformas de hardware, RTOS, y soluciones basadas en FPGA.

\section{TRABAJOS FUtUROS}

Utilizando como base el presente proyecto se presenta una lista de líneas de trabajo en el campo de diseño de SoCs que se recomiendan abordar. Estas son:

- Buses PCI con IP Core PCIPLBv46 RC/EP y Bridge for PCI Express

- $\quad$ Networking con IP Core XPS LL TEMAC.

- $\quad$ Sistema con dos procesadores utilizando IP Cores XPS Mailbox y XPS Mutex.

- Uso de protocolos de comunicación: Bus CAN, Ethernet Industriales, USB entre otros.

- Sistema que incluya IP Cores creados por el usuario.

- Depuración de hardware con ChipScope Pro

- Profundizar en el uso de Xilkernel

- Estudio de otros RTOS como Petalinux.

- Implementar técnicas de control adaptativo, difuso, o por redes neuronales en un SoC.

- Desarrollar control y monitoreo de procesos con HMI sobre un SoC.

- Estudio de la arquitectura AMBA de ARM.

\section{RECONOCIMIENTO}

$\mathrm{Al}$ ingeniero Byron Navas, por ser el mentor de este proyecto y la persona guía durante la etapa inicial. 


\section{BIBLIOGRAFÍA}

[1] MARTIN, grant y CHANG, henry, Winning the SoC RevolutionExperiences in Real Design, Kluwer Academic Publisher, Estados Unidos 2003, 311 páginas.

[2] NAVAS, Byron, Chips Diseñados en Ecuador, Revista E-Ciencia ESPE, Edición 2, Diciembre 2009

[3] MARTIN, grant y CHANG, henry, Surviving the SoC RevolutionA Guide to Platform - Based Design, Kluwer Academic Publisher, Estados Unidos 1999, 235 páginas.

[4] XILINX, Inc., Getting Started with the Spartan-6 FPGA SP605 Embedded Kit, documento UG727 (v1.1), June 21, 2010.

[5] XILINX, Inc., Hardware and Demonstration Setup Guide, documento UG526 (v1.4), Septiembre 24, 2009.

[6] XILINX, Inc., EDK Concepts, Tools, and Techniques, documento UG683, 2009. 